\title{
ACTIVIDADES INTERDISCIPLINARIAS
}

\author{
Susana María Nolasco \\ Departamento de Ingeniería Química, Facultad de Ingeniería, Universidad Nacional del Centro de la Provincia de Buenos Aires,
} Avda del Valle 5737, B7400JWI-Olavarría, Provincia de Buenos Aires - Argentina

Recebido em 29/3/01; aceito em 24/10/01

INTERDISCIPLINARY ACTIVITIES. The objective of this work is to show both experiences of multidisciplinary activities and the way to an interdisciplinary vision of the development of scientific - technological investigations carried out at Engineering Faculty of the Universidad Nacional del Centro de la Provincia de Buenos Aires, under the investigation program "Seed Technology (TECSE)". Interdisciplinary teamwork is an inevitable quality in the XXI Century scientist or professional. Therefore a qualitative characteristic is imposed from a multidisciplinary work stage towards an interdisciplinary teamwork activity, turning a narrow vision of problems from a particular profession or science into an articulation of knowledge and interests to obtain an integral solution.

Keyword: interdisciplinary activities; teamwork.

\section{INTRODUCCION}

"Desde muy temprana edad nos enseñan a analizar los problemas, a fragmentar el mundo. Al parecer esto facilita las tareas complejas, pero sin saberlo pagamos un precio enorme. Ya no vemos las consecuencias de nuestros actos; perdemos nuestra conexión con una totalidad vasta. Cuando intentamos ver la "imagen general", tratamos de ensamblar nuevamente los fragmentos, enumerar y organizar todas la piezas. Pero... esta tarea es fútil: es como ensamblar los fragmentos de un espejo roto para ver un reflejo fiel. Al cabo de un tiempo desistimos de tratar de ver la totalidad".

P.M. Senger ${ }^{1}$

En un mundo signado por la globalización, el acelerado desarrollo científico-tecnológico y de las comunicaciones, más que nunca es necesario desterrar una visión de un mundo compuesto por fuerzas separadas y desconectadas. Debemos admitir que no se puede comprender un todo estudiando aisladamente sus partes, fuera de su contexto.

La intedisciplinariedad es la forma más adecuada para el desarrollo tecnológico y científico, llevando implícita una compleja articulación de los conocimientos de diferentes campos y orientaciones científicas y productivas ${ }^{2}$.

"La creación de una masa crítica en ciencia debe superar la visión estrecha de concebirla como intercambios entre especialistas sobre un tema para pasar a su verdadero concepto: las interacciones de los científicos entre si y de estos con los componentes de la sociedad"3.

El éxito de las actividades profesionales o científicas dependen, cada vez más, de la tarea grupal y de la performance del conjunto de sus integrantes. Equipos interdisciplinarios que buscan construir una visión compartida de las problemáticas, que emprenden un aprendizaje conjunto que lleve al desarrollo de las aptitudes de los integrantes del grupo bajo perspectivas que trasciendan lo individual.

El objetivo del presente trabajo es presentar experiencias de actividades multidisciplinarias y el pasaje hacia una visión

\footnotetext{
*e-mail: snolasco@fio.unicen.edu.ar
}

interdisciplinaria del desarrollo de investigaciones científicotecnológicas, llevadas a cabo en la Facultad de Ingeniería de la Universidad Nacional del Centro de la Provincia de Buenos Aires, en el marco del Programa de Investigación: “Tecnología de Semillas (TECSE)"

\section{DESARROLLO}

\section{Ambito de desarrollo de las investigaciones}

El programa de Investigación "TECNOLOGIA DE SEMILLAS (TECSE)" se desarrolla en la Facultad de Ingeniería de la Universidad Nacional del Centro de la Provincia de Buenos Aires (Olavarría, Argentina) y surgió en el año 1993 de la unión de distintas líneas de investigación que se desarrollaban en dicha Facultad, dedicadas a la caracterización morfológica, física y química de semillas o granos de diferentes especies vegetales y al estudio del almacenamiento y acondicionamiento de cereales y oleaginosos.

\section{Objetivo del programa}

El objetivo de este proyecto es la formación de un grupo interdisciplinario de investigadores que oriente su estudio al mejoramiento de las condiciones de producción, postcosecha y procesamiento de cereales y oleaginosos; así como a la búsqueda de nuevas fuentes seminales de productos alimenticios y/o industriales.

Teniendo en cuenta que,

$\checkmark$ Argentina produce un espectro casi completo de granos (cereales y oleaginosos),

$\checkmark$ La industrialización y exportación de cereales y oleaginosos y sus subproductos constituyen un sector de suma importancia y creciente expansión en nuestro país,

$\checkmark$ El mercado internacional evoluciona hacia una demanda cada vez mas exigente en cantidad y calidad de los productos, lo que obliga a realizar todos los esfuerzos necesarios para poder disponer de granos, cada vez de mayor calidad y con los medios adecuados para conservarla,

se plantea este Programa de Investigación con el objeto de generar información, conocimientos y tecnología. 


\section{Organización del programa}

El desarrollo del mismo se organiza en dos área inter-relacionadas:

\section{Area A. MATERIA PRIMA: GRANOS}

Objetivo general: Estudiar las características morfológicas, físicas y químicas de semillas o granos de diferentes variedades vegetales y los efectos que sobre la calidad de los mismos ejercen factores genéticos, ambientales y procesos tecnológicos.

Proyectos: *Tecnología de Oleaginosos. *Estudio químico integral y aprovechamiento industrial de semillas de malezas de la zona de Olavarría.

Area B. ACONDICIONAMIENTO Y ALMACENAMIENTO DE CEREALES Y OLEGINOSOS

Objetivo general: Estudiar los procesos de acondicionamiento y almacenamiento de granos a fin de aportar conocimientos que permitan garantizar la calidad e inocuidad de los mismos y de sus subproductos, con el menor costo posible y reduciendo al mínimo las pérdidas postcosecha.

Proyectos: * Secado de granos. *Aireación de silos. * Monitoreo y adquisición de datos en un silo de granos.

\section{Actividades multidisciplinarias}

$\checkmark$ En el marco del proyecto "Estudio químico integral y aprovechamiento industrial de semillas de malezas de la zona de Olavarría" se estudian las características morfológicas, físicas y químicas de semillas de especies inexplotadas que crecen en el suelo argentino, especialmente de la zona de Olavarría y su probable aprovechamiento industrial (alimentación, pinturas, aceites esenciales, aditivos, etc.). Fueron analizadas semillas de especies pertenecientes diferentes familias ${ }^{4,5}$ contando con aportes especialmente de las disciplinas: Agronomía, para la identificación especies, ubicación de zona de desarrollo, cosecha de semillas, estudios desde el punto de vista de la nutrición animal (aporte de profesionales de diferentes Facultades de Agronomía del país y organismos estatales relacionados); Ciencias Exactas y Naturales en lo que se refiere a aspectos químicos, bromatológicos y microbiológicos (contando con la colaboración de la Facultad de Ciencias Exactas y Naturales, Departamento de Química Orgánica de la Universidad de Buenos Aires).

$\checkmark$ El manejo de los granos luego de la cosecha es una práctica que cada día adquiere mayor importancia en países, como Argentina, con marcada inclinación agrícola. El manejo postcosecha de los granos es una actividad que se puede caracterizar como multidisciplinaria, en ella se aplican principios de las ciencias biológicas, física, matemática y química, conjuntamente con los correspondientes a economía y relaciones humanas, conjugándose la participación de diversas disciplinas, tales como: ingeniería química, agronomía, electromecánica, electrónica. En esta temática se hizo especial hincapié en los aspectos referidos a ingeniería de procesos tales como:

- Aireación de silos: sistemas utilizados para forzar aire a través de la masa de granos, permitiendo mejorar las condiciones de almacenamiento y disminución de riesgo del deterioro del material. El éxito de esta operación depende el adecuado diseño de estos sistemas que requieren información concerniente a la resistencia al flujo de aire de los granos ${ }^{6,7}$.

- Secado de granos: determinar experimentalmente las curvas de secado e interpretarlas con modelos preferentemente difusivos, con el objeto de obtener la información cinética necesaria para su aplicación en modelos de lecho alto de secadoras continuas ${ }^{8,9}$.

- Monitoreo y adquisición de datos en un silo de granos: cuya finalidad es obtener información que oriente o conduzca el proceso de ventilación de silos y secado de granos a baja temperatura. A partir de estos datos, modelizar la evolución de la temperatura tanto en la pared de un silo como en el lecho de grano, analizando el efecto de las fluctuaciones de temperatura sobre la calidad del grano ${ }^{10,11}$. Para el desarrollo de estos estudios se requirió de equipamiento electrónico (sistemas de control con hardware y software específicos diseñados para el monitoreo y adquisición de dato de tres variables fundamentales: presión diferencial, temperatura y humedad); lo que derivó en el desarrollo de una actividad multidisciplinaria con los profesionales del Departamento de Electromecánica de nuestra Facultad de Ingeniería (área electrónica), quienes desarrollaron parte del equipamiento mencionado.

Es incipiente una actividad interdisciplinaria en este Area (Acondicionamiento y almacenamiento de cereales y oleaginosos), que conduzca al análisis del almacenamiento y acondicionamiento de los granos como un todo, abarcando los aspectos biológicos, químicos, agronómicos, de procesos, electrónicos, económicos y de relaciones humanas. En el marco del secado de granos, donde la situación económica imperante exige una mejora en la competitividad y requiere maximizar la eficiencia de los procesos, se plantea un plan de investigación e innovación, tendiendo a integrar la faceta científica-tecnológica con los aspectos económicos, comerciales y sociales de la problemática.

\section{... Hacia actividades interdisciplinarias}

La Facultad de Ingeniería de la Universidad Nacional del Centro de la Provincia de Buenos Aires (a través de Grupo de Investigación "Tecnología de Semillas -TECSE-") y la Facultad de Ciencias Agrarias de la Universidad de Mar del Plata (con la participación del Grupo de Investigación "Fisiología Vegetal"), desde 1995 desarrollan el Programa de Investigación "Estudio de la calidad de los frutos de girasol; aceites y subproductos: efectos de factores genéticos y ambientales"; con un enfoque interdisciplinario, tendiendo a la integración de la visión del agrónomo con la del químico e ingeniero químico, respecto a los factores y procesos que influyen en la calidad de los frutos de girasol, aceite y subproductos. Conocer la influencia que factores genéticos y ambientales ejercen sobre dicha calidad permitirá adoptar estrategias productivas que optimicen la rentabilidad del cultivo. El conocimiento de las características de las semillas productoras de aceite, en particular aquellas que afectan, directa o indirectamente el proceso tecnológico de producción de aceites y harinas, contribuye al control de la materia prima que ingresa al/los procesos, factor fundamental dentro del desarrollo de la agroindustria ${ }^{12,13}$.

En este marco, se desarrollaron diferentes investigaciones, entre las que podemos mencionar:

$\checkmark$ Estudio y modelización del efecto de la radiación solar(PAR) interceptada por la planta sobre el porcentaje de aceite de los frutos y su calidad,

$\checkmark$ Explorar la variabilidad entre híbridos, regiones y años de características que definen la calidad de los frutos y que determinan en gran medida la cantidad de aceite que puede extraerse, la calidad del aceite y de los subproductos, relacionándolas con variables climáticas de fácil medición,

$\checkmark$ Evaluación y modelación del efecto de la densidad de siembra sobre características físicas y químicas de los frutos del girasol, 
$\checkmark$ Tocoferoles en aceite de girasol: variabilidad genética y variabilidad ambiental, relación con otras variables de calidad del fruto,

$\checkmark$ Aptitud al descascarado de frutos de girasol: Influencia de factores genéticos y ambientales- Innovaciones agronómicas y tecnológicas. Es éste un proyecto interdisciplinario mucho más ambicioso que los anteriores, involucra la interacción entre profesionales de variadas especialidades, tales como: química, genética, agronómica, procesos, morfología vegetal, resistencia de materiales.

- Dificultades que se debieron afrontarse:

$\checkmark$ Conjugar conceptos, terminologías, metodologías, puntos de vistas de diferentes disciplinas; lo que requirió de un gran esfuerzo y predisposición de los investigadores involucrados para pasar de la visión de "intercambio" a la de "interacción",

$\checkmark$ Paso de la "discusión" (planteo de ideas en una competencia donde el ganador se queda con todo) al " diálogo" (capacidad de los miembros del equipo para ingresar en el auténtico "pensamiento conjunto"). Para los griegos, dia-logos, significaba el libre flujo del significado a través del grupo, lo cual permite al grupo descubrir percepciones que no se alcanzan individualmente,

$\checkmark$ Lograr una fluida comunicación entre los investigadores, afectada por la distancia existente entre los establecimientos involucrados (aproximadamente $250 \mathrm{~km}$.). El acceso a los medios de comunicación por red informática (INTERNET, correo electrónico, etc.) dotó al equipo de una herramienta ágil para optimizar el desarrollo de las actividades. Ya no fue necesario la coordinación de numerosos viajes con las consiguientes demoras temporales y costos correspondientes.

- Metas alcanzadas:

$\checkmark$ La configuración de un equipo capacitado para el abordaje efectivo de la complejidad, con un enfoque integrador de las problemáticas bajo estudio, aprendiendo a crecer en conjunto e incrementando la percepción, creatividad e innovación de todos sus integrantes.

$\checkmark \quad$ El fructífero resultado de esta actividad interdisciplinaria se ve reflejado en los numerosos trabajos presentados en Congreso, publicaciones científicas y de divulgación, así como en el desarrollo de actividades de transferencia hacia el medio productivo.

La calidad de los frutos, aceites y subproductos de girasol, depende de factores genéticos, ambientales, prácticas de cultivo, así como de las condiciones de cosecha, postcosecha y procesamiento. Conocer la relaciones entre estas etapas permitirá obtener productos de mejor calidad, optimizar procesos y mejorar aspectos económicos y comerciales, sociales y humanos. Como se había mencionado con anterioridad, no se puede comprender un todo estudiando aisladamente sus partes.

\section{CONCLUSIONES}

Los desafíos que nos presenta el nuevo milenio no escapan de la necesidad del trabajo en equipo de grupos interdisciplinarios. Los problemas actuales y futuros ya no se podrán abordar bajo la visión de una sola disciplina o profesión. La habilidad de trabajar en equipo bajo un enfoque interdisciplinario es un rasgo inevitable en el científico y el profesional del siglo XXI. Es por ello que, se impone un salto cualitativo, desde una etapa de trabajo multidisciplinario hacia una actividad interdisciplinaria donde los profesionales y científicos que se reúnen en equipo dejan de lado una visión estrecha de los problemas a partir de la óptica de una profesión y/o ciencia en particular, dando paso a una compleja articulación de conocimientos e intereses que permitan obtener una solución integral de los problemas.

\section{REFERENCIAS}

1. Senger, P.M.; La quinta disciplina, Editorial Juan Graneca S.A., 1995.

2. Gogorza, L.; Morán, P.; Torres, J.; Di Santo, M.; Segundo Congreso Mundial de Educación Internacional, Integración y Desarrollo, Buenos Aires: Argentina, 1999.

3. Armenteros Acosta, M. del C.; Vega García C.; Congreso Iberoamericano de Enseñanza de la Ingeniería y la Arquitectura. La Habana: Cuba, 1999.

4. Nolasco, S.M.; Quiroga, O.E.; Wiese, B. ; Vigo, S.M.; Universidad Abierta, Encuentro de Investigadores, Universidad Nacional del Centro de la Provincia de Buenos Aires: Argentina, 1998.

5. Nolasco, S.M.; Quiroga O.E.; Wiese, B.; Vigo, S.M.; Acta Horticulturae (ISHS) 1999, 501, 209.

6. Pagano, A.M.; Crozza, D.E.; Nolasco S.M.; Universidad Abierta, Encuentro de investigadores, Universidad Nacional del Centro de la Provincia de Buenos Aires: Argentina, 1998.

7. Pagano, A.M.; Crozza, D.E.; Nolasco S.M.; J. Am. Oil Chem. Soc. 1998, 75,30 .

8. Gely, M.C.; Giner, S.A.; Universidad Abierta, Encuentro de Investigadores, Universidad Nacional del Centro de la Provincia de Buenos Aires: Argentina, 1998.

9. Gely, M.C.; Giner, S.A.; Drying Technology 2000, 18, 1449.

10. Crozza, D.E.; Pagano, A.M.; Universidad Abierta, Encuentro de Investigadores, Universidad Nacional del Centro de la Provincia de Buenos Aires: Argentina, 1998.

11. Pagano, A.M.; Crozza, D.E.; Drying Technology 2000, 18, 1081.

12. Nolasco, S.M.; Riccobene I.C., Santalla E.M., Garay, M.; Universidad Abierta, Encuentro de Investigadores, Universidad Nacional del Centro de la Provincia de Buenos Aires: Argentina, 1998.

13. Nolasco, S.M.; Dosio, G.A.A.; Riccobene, I.C.; Santalla, E.M.; Aguirrezábal, L.A.N.; Proceedings of the $15^{\text {th }}$ International Sunflower Conference, Tolouse: Francia, 2000, vol. 1. 